\title{
Collaborer par le biais du portfolio numérique pour améliorer l'accompagnement du stagiaire : bilan au terme de deux années d'expérimentation
}

Par Nadia Cody, Nicole Monney, Roxanne Labrecque et Caroline Boisvert, Université du Québec à Chicoutimi

\section{Résumé}

Au Québec, les fonctions de l'enseignant associé et du superviseur universitaire sont balisées par un référentiel de compétences (Portelance, Gervais, Lessard et Beaulieu, 2008), lequel a fait l'objet d'études (L'Hostie, Cody, Cividini, Allaire et Gauthier, 2008-2011 ; Cody, Cividini et L'Hostie, 2011-2013) démontrant que ces acteurs clés souhaitent collaborer plus efficacement et aider le stagiaire à établir des liens entre les savoirs formels et expérientiels en formation initiale. Afin de répondre à ce besoin, une équipe composée de chercheurs universitaires, d'enseignants associés et de superviseurs universitaires a repensé l'usage du portfolio numérique pour en faire un outil d'accompagnement et de collaboration. Des données issues d'un questionnaire $(n=19)$ et de deux focus groups $(n=8)$ ont été analysées du point de vue de la collaboration entre les enseignants associés et les superviseurs universitaires. L'article mettra en lumière les résultats obtenus au terme de deux années d'expérimentation, notamment l'impact du dispositif sur la collaboration entre ces deux catégories d'intervenants.

Mots clés: portfolio numérique, collaboration, enseignant associé, superviseur, accompagnement, stagiaire 


\section{REVUE HYBRIDE DE L'ÉDUCATION}

\section{Problématique}

Dans le cadre du processus de supervision des stages en milieu scolaire, l'enseignant associé (EA) et le superviseur universitaire (SU) exercent, en collaboration, des fonctions complexes balisées par un référentiel de compétences (Portelance, Gervais, Lessard et Beaulieu, 2008). Des études régionales, menées au cours des dernières années (L'Hostie, Cody, Cividini, Allaire et Gauthier, 2009-2012'1; Cody, Cividini et L'hostie, 2011-2013²), ont mis en évidence que ces intervenants rencontrent des difficultés et identifient des besoins en lien avec certaines compétences de ce référentiel dont (a) celle relative à l'accompagnement du stagiaire dans le développement des compétences professionnelles requises pour l'exercice de la profession enseignante et (b) celles axées sur sa pratique réflexive et sur l'élaboration de liens entre les savoirs formels et expérientiels (approche intégrative). Selon Martin (2002), le stagiaire a de la difficulté à réaliser ces liens; d'entrée de jeu, il accorde plus d'importance aux savoirs issus de l'expérience qu'à ceux acquis en milieu universitaire. Des recherches menées en formation initiale (Perrenoud, 2001; Cody, 2013) démontrent pourtant que le développement des compétences en enseignement doit résulter tant d'une théorie associée à la pratique que d'une pratique découlant d'une théorie, qu'il ne suffit pas de juxtaposer des cours à teneur plus théorique et des activités de formation pratique, pas plus d'ailleurs qu'il ne suffit de structurer la formation sous la forme d'une alternance entre l'université et le milieu scolaire (Gagnon, Mazalon et Rousseau, 2010). Pour bien accompagner le stagiaire dans le développement des 12 compétences (Portelance, 2009) et dans son analyse réflexive (Schön, 1996), l'EA et le

\footnotetext{
1 L'Hostie, M., Cody, N., Cividini, M., Allaire, S. et Gauthier, R. (2009-2012). Renforcer le savoir d'expérience, les compétences et la professionnalité des enseignants associés à travers une recherche-action-formation réalisée en communauté de pratique. Projet de recherche financé par le ministère de l'Éducation, du Loisir et du Sport (MELS), Chantier 7.

2 Cody, N., Cividini, M. et L'Hostie, M. ((2011-2013). Les stratégies de collaboration favorisées par les enseignants associés et les superviseurs universitaires dans la formation des stagiaires : rôles communs et complémentaires de ces deux acteurs clés. Projet de recherche financé par le Consortium de recherche en éducation (CRRE) et par la Fondation de l'Université du Québec à Chicoutimi (FUQAC).
} 


\section{REVUE HYBRIDE DE L'ÉDUCATION}

SU, coresponsables de la formation en milieu de pratique, doivent l'aider à établir ces liens entre les savoirs formels et expérientiels. Cela nécessite que ces deux acteurs clés travaillent en étroite collaboration, par le biais de la réflexion et du dialogue, et qu'ils s'appuient l'un sur l'autre afin d'étayer leur contribution respective (Pharand et Boudreault, 2011). Or, cette collaboration peut parfois être difficile à établir en raison des limites de temps et du manque de connaissances et d'outils qui leur permettraient d'être au fait des apprentissages réalisés durant les cours universitaires et lors des stages (Gervais, 2008).

Un dispositif reconnu comme outil d'analyse réflexive est le portfolio de développement professionnel (Bibeau, 2007; Labelle, 2015). ॥ s'agit d'une collection des meilleurs travaux documentés par l'enseignant qui devrait être co-construite en processus continu à l'aide de mentors ou de collègues (Wolf, 1996). Dans le cas du stagiaire, ces travaux sont puisés à la fois dans les cours et les stages. L'EA et le SU jouent ainsi le rôle de mentors. À ce jour, la recherche a surtout documenté l'apport du portfolio au développement professionnel du futur enseignant et particulièrement l'analyse réflexive qui en résulte (Bibeau, 2007; Goupil, 1998; Naccache, Samson et Jouquan, 2006). Peu d'études ont porté sur la façon dont l'EA et le SU pourraient l'exploiter afin de favoriser leur collaboration et d'améliorer l'accompagnement du stagiaire (Zeichner et Hutchinson, 2004). Pour faciliter l'accès au portfolio, la présente étude intitulée Mise en place d'un dispositif hybride facilitant l'accompagnement et la collaboration dans le cadre de la supervision des stages de première année du baccalauréat d'éducation au préscolaire et d'enseignement au primaire et d'une durée de deux ans (2014-2016), s'est proposée de concentrer la réflexion autour d'un format numérique qui permet à l'EA et au SU de collaborer en présentiel et à distance dans l'accompagnement du stagiaire.

L'objectif général de la recherche consistait notamment à dégager les avantages et les difficultés associés à ce dispositif sur le plan de l'accompagnement et de la collaboration entre les deux acteurs clés. Cet 


\section{REVUE HYBRIDE DE L'ÉDUCATION}

article présente les résultats de l'implantation du portfolio numérique dans

le cadre du stage de première année du baccalauréat d'éducation au préscolaire et d'enseignement au primaire de I'UQAC au terme de deux années d'expérimentation. II vise, entre autres, à présenter la structure du dispositif hybride (Petit, 2016) qui favorise la collaboration entre l'EA et le $\mathrm{SU}$ et à identifier les modalités de collaboration associées à ce même dispositif.

\section{Cadre conceptuel}

Pour répondre à l'objectif général de la recherche, deux concepts essentiels sont définis : le dispositif hybride et la collaboration.

Un dispositif hybride se caractérise par la présence de dimensions innovantes liées à la mise à distance (Charlier, Deschryver et Peraya, 2006). Dans le cas des stages, le dispositif de base comprend des acteurs (SU, EA et stagiaire), des objectifs à atteindre pour s'assurer du développement des compétences et des modalités d'accompagnement et de collaboration (nombre de journées en stage, nombre d'observations par le SU, travaux à remettre, responsabilités de l'EA, responsabilités du $\mathrm{SU}$, etc.). Dans le cas de cette recherche, un élément est ajouté à ce dispositif de base, à savoir, le portfolio numérique. Par conséquent, un dispositif de formation devient hybride lorsqu'il utilise un environnement technopédagogique (portfolio numérique) et repose sur des formes complexes de médiatisation et de médiation (Charlier et al., 2006).

La médiatisation est le processus de scénarisation de contenus sous diverses formes telles que l'information, l'interaction, la réalisation de tâche, l'autoévaluation, l'accompagnement, etc. (Peraya, 2014). Dans notre cas, la médiatisation comprend les différentes tâches demandées au stagiaire et pour lesquelles il doit produire des pages dans son portfolio numérique. La médiation, quant à elle, est la position occupée par le côté humain dans le dispositif (Peraya, 2010), à savoir les interactions entre le stagiaire, l'EA le SU. Le portfolio numérique fait partie du dispositif hybride de stage et permet de médiatiser les contenus 


\section{REVUE HYBRIDE DE L'ÉDUCATION}

d'apprentissage pour favoriser le développement des compétences, mais aussi, pour donner une place à la médiation entre les différents acteurs. Par exemple, le stagiaire doit rédiger une réflexion à partir de trois questions initiales pour définir son identité professionnelle, créer des planifications de situations d'enseignement-apprentissage, réaliser un portrait de la classe, etc. Cette scénarisation vise la construction d'une expertise de l'étudiant à partir des savoirs formels et des savoirs expérientiels, et ce, pour orienter ses actions et ses interventions professionnelles (Bibeau, 2007). Le SU et l'EA prennent connaissance des différentes réalisations du stagiaire et peuvent commenter le tout par le biais de la plateforme du portfolio numérique. Ainsi, le portfolio supporte la planification des apprentissages, l'évaluation formative et la démarche réflexive (Naccache et al., 2006). II joue le rôle de médiateur entre le stagiaire et les formateurs puisqu'il permet l'interaction entre les différents acteurs. La Figure 1 présente la structure du dispositif hybride de stage intégrant le portfolio numérique et les concepts de médiatisation et de médiation.

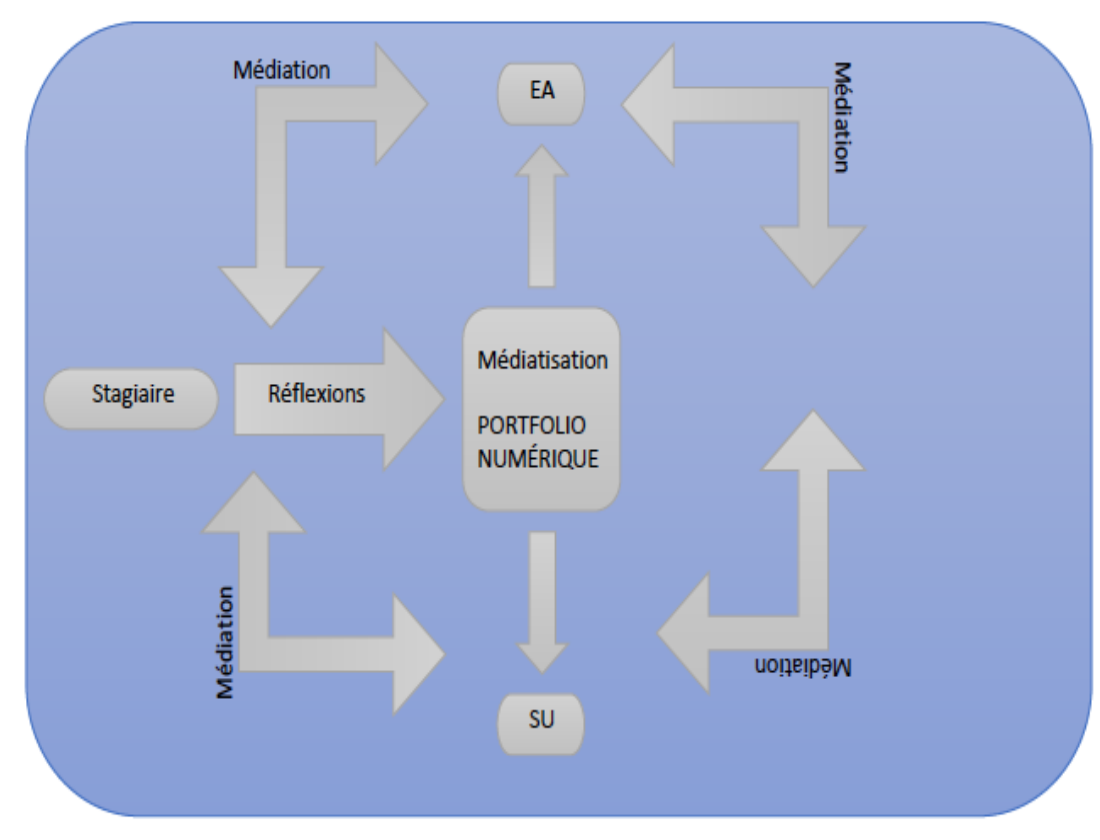




\section{REVUE HYBRIDE DE L'ÉDUCATION}

Figure 1. Dispositif hybride : l'intégration du portfolio numérique dans le dispositif de stage

Cette étude s'intéresse plus particulièrement à l'exploitation que pourrait en faire l'EA et le SU. Ainsi, l'analyse du dispositif met en évidence les modalités de collaboration permettant le développement des compétences chez le stagiaire. Selon Dionne et Savoie-Zajc (2011), la collaboration est un lieu ou un espace professionnel dans lequel des individus peuvent s'exprimer et entreprendre une démarche conjointe. $\mathrm{Ce}$ concept est polysémique et comme le propose la Figure 2, il peut prendre différentes formes selon le degré de relation, d'engagement et de consensus existant entre les intervenants.

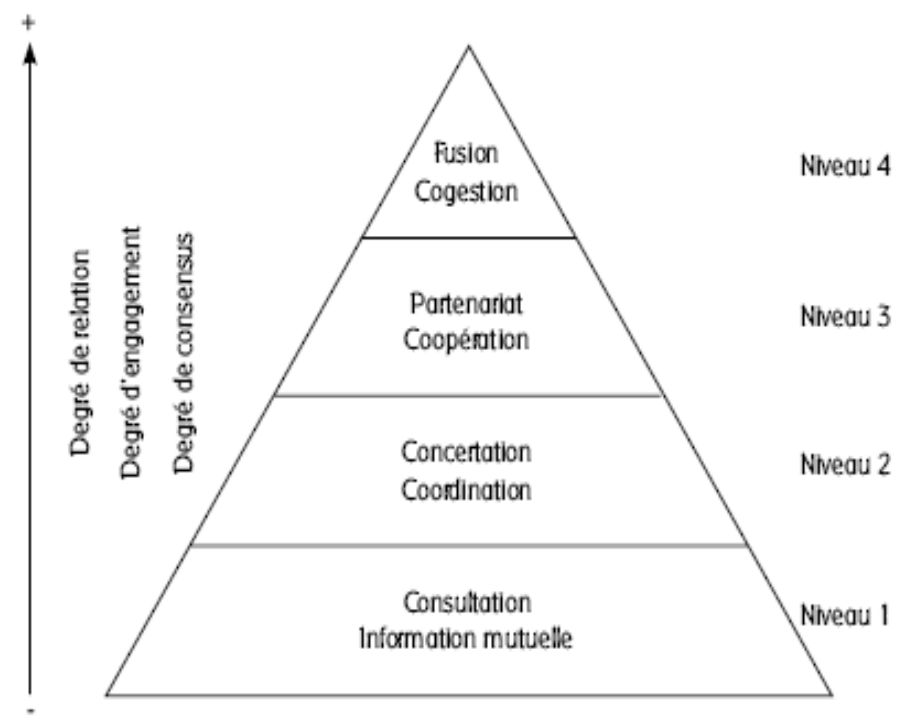

Figure 2. Les diverses formes de collaboration (Larivée, 2003 cité par Larivée, Kalubi et Terrisse, 2006)

Selon Larivée, Kalubi et Terrisse (2006), la collaboration peut, dans un premier niveau, être uniquement une forme de consultation. Dans ce cas, l'EA et le SU s'échangent de l'information mutuelle sur ce qu'ils ont chacun observé du stagiaire. Le niveau 2 de la collaboration est 


\section{$\&$}

\section{REVUE HYBRIDE DE L'ÉDUCATION}

davantage de l'ordre de la concertation durant laquelle l'EA et le SU vont se coordonner sur les objectifs à atteindre. Cependant, chacun y va avec les actions qu'il juge opportunes. Par exemple, les formateurs peuvent viser comme objectif une meilleure gestion de classe de la part du stagiaire. L'EA pourrait proposer au stagiaire d'utiliser ses propres stratégies (tableau de motivation, signal de retour au calme, etc.), alors que de son côté, le SU suggérerait au stagiaire de retourner dans ses notes de cours relatives à la gestion de classe. La responsabilité d'évaluer cette compétence au terme du stage est néanmoins attribuée au SU. Le concept de partenariat, qui correspond au niveau 3 du modèle de Larivée (2003, cité par Larivée et al., 2006), se définit sous la forme d'une relation de collaboration avec une vision partagée, ce qui exige que le but soit commun et tienne compte des compétences respectives de chaque partenaire (Gervais, 2008). Dans le cas du EA et du SU, les compétences ciblées sont celles du Cadre de référence pour la formation des formateurs de stagiaires (Portelance et al., 2008). En d'autres termes, dans le cadre d'un partenariat, les intervenants s'engagent dans la poursuite d'un but commun en optant pour une position d'ouverture aux savoirs et aux expériences des autres (Portelance, Pharand et Borges, 2011). Finalement, le niveau 4 cible la cogestion et la fusion entre les intervenants. À ce niveau, l'expertise des intervenants ne fait plus qu'une et tous les objectifs se réalisent en cogestion. II s'agit là d'un niveau de collaboration extrême qui demanderait au SU de passer pratiquement le même temps en classe que l'EA. D'un point de vue purement administratif, il est difficile, voire même impossible, de penser que cela soit réalisable.

\section{Méthodologie}

Cette section présente le contexte du premier stage (contexte dans lequel cette recherche a été réalisée), le type de recherche, les collaborateurs et les participants de même que la saisie, le traitement des données et l'analyse du matériel empirique. 


\section{REVUE HYBRIDE DE L'ÉDUCATION}

\section{Contexte du stage I}

Le stage I se déroule sur 23 jours (trois journées perlées et 20 jours consécutifs). Le stagiaire doit réaliser différentes tâches durant son stage dont une réflexion sur son identité professionnelle, des planifications de situations d'enseignement, des réflexions portant sur les liens entre les cours et les stages, le portrait du groupe-classe et le portrait des élèves. Dans le cadre du dispositif de formation habituel, le stagiaire remettait ces différents travaux à son SU, et ce, sans que l'EA n'ait la responsabilité de valider ces derniers. Avec l'intégration du portfolio numérique, tous les travaux sont déposés sur la plateforme et I'EA reçoit une copie du lien Web. II est donc fortement invité à consulter les travaux, les commenter et valider leur contenu. II a fallu ainsi se questionner sur les étapes de la mise en place de ce nouveau dispositif qui modifie les rôles de chacun en ce qui concerne la consultation des différents travaux. C'est la raison pour laquelle nous avons opté pour l'intégration des différents formateurs dans l'équipe de recherche.

Type de recherche, collaborateurs et participants

En mettant à contribution différentes expertises, provenant à la fois des milieux scolaire et universitaire, pour développer le dispositif hybride, ce projet s'inscrit dans le modèle de la recherche participative, laquelle "va dans le sens de valoriser un processus de production des connaissances réalisé de concert avec les acteurs concernés " (Anadon, 2007, p. 3). L'équipe était composée de deux chercheuses de l'UQAC, de deux EA, de deux SU, agissant à titre de professeurs invités en prêt de service (PIPS), d'une professionnelle de recherche, spécialiste du changement assisté, et de deux étudiantes de premier cycle oeuvrant comme assistantes de recherche. Les membres de l'équipe de recherche se sont réunis à quatre reprises la première année pour identifier les modalités d'accompagnement et de collaboration intégrant le portfolio numérique, structurer le dispositif hybride, identifier des participants pour le projet pilote, planifier l'intégration du dispositif et établir un premier bilan 


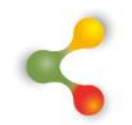

\section{REVUE HYBRIDE DE L'ÉDUCATION}

des avantages et des difficultés liés à son intégration. La deuxième année, quatre rencontres ont aussi été tenues, selon des dispositions identiques à celles de l'an 1, pour améliorer les modalités d'accompagnement et de collaboration en lien avec le portfolio numérique, affiner la structure du dispositif hybride, planifier son intégration (formation des $\mathrm{EA}$, des $\mathrm{SU}$ et des stagiaires de première année) et établir le bilan final des avantages et des difficultés qui y sont rattachés. La première année a été consacrée à la mise sur pied du projet pilote (auprès de cinq stagiaires, cinq EA et deux $\mathrm{SU}$ ), alors que la deuxième année visait à intégrer le dispositif à plus large échelle auprès de tous les stagiaires de première année $(n=58)$ ainsi que des EA ( $n=$ $58)$ et des SU $(n=8)$.

Saisie, traitement des données et analyse du matériel empirique

À la toute fin de la deuxième année du projet, un questionnaire a été distribué aux EA qui accompagnaient les stagiaires. Une analyse descriptive a été effectuée à partir des questionnaires puis deux focus groups ont été menés auprès des SU. Une analyse de contenu (Bardin, 1989), réalisée à partir du verbatim, a permis de dégager des éléments en lien avec la structuration du dispositif et les modalités de collaboration mises en oeuvre par les EA et les SU. L'ensemble des éléments recensés a été analysé du point de vue des concepts de dispositif hybride et de collaboration.

\section{Présentation des résultats}

Les résultats obtenus sont présentés en fonction de deux objectifs spécifiques de l'étude qui font l'objet du présent article soit (a) structurer le dispositif afin de favoriser la collaboration entre l'EA et le SU et (b) identifier les modalités de collaboration associées au portfolio numérique de développement professionnel. Dix-neuf EA ont répondu au questionnaire et tous les SU ont participé à l'un ou l'autre des focus groups. 


\section{REVUE HYBRIDE DE L'ÉDUCATION}

\section{Un dispositif structuré afin de favoriser la collaboration entre les EA et les SU}

Le point de vue des SU

Les résultats montrent que l'appropriation de la structure demeure un facteur déterminant quant à la motivation des acteurs envers l'utilisation du dispositif en vue de collaborer. La plupart des SU affirment que le fonctionnement de la plateforme n'est « pas compliqué ». Bien que certains d'entre eux recommandent d'ajouter un soutien technique ou d'améliorer la formation, les tutoriels et les formations ont été selon eux « aidants ». Or, force est de constater que certains participants ont connu des difficultés techniques qui ont limité le rendement et l'efficacité d'une collaboration, soit par un manque de compétences (deux SU) ou par un manque d'intérêt (un SU). Les résultats font voir que cinq SU ont éprouvé des difficultés à gérer certaines options de commentaires, notamment sur le plan des modifications, de création de pages et de partage de liens. Un SU doute du fait que les EA consultent les outils mis à leur disposition comme le tutoriel d'utilisation et deux d'entre eux s'entendent sur le fait que l'utilisation de la plateforme exige un temps supplémentaire d'appropriation.

\section{Le point de vue des EA}

À la question de savoir quelle est l'expérience générale vécue par les EA par rapport à l'utilisation du portfolio, 14 enseignants se disent favorables. L'outil est facile, accessible et pertinent. II offre la possibilité de connaitre le stagiaire et de conserver des traces tout en permettant un suivi des travaux. Trois enseignants considèrent que l'appropriation de l'outil est plutôt difficile. Un seul enseignant affirme cependant avoir une préférence pour la version papier. Un enseignant n'a pas répondu à la question.

Les modalités de collaboration intégrant le portfolio numérique de développement professionnel 


\section{8}

\section{REVUE HYBRIDE DE L'ÉDUCATION}

Les résultats relatifs aux modalités de collaboration intégrant le portfolio numérique de développement professionnel sont divisés en deux sous-thèmes : collaborer par le biais d'un outil interactif et collaborer pour une vision commune afin d'améliorer la qualité de l'accompagnement. Les résultats de cette étude révèlent que le portfolio numérique favorise la collaboration entre les EA et les SU en raison de la possibilité, pour ces deux acteurs, de commenter les différents travaux des stagiaires.

\section{Collaborer par le biais d'un outil interactif}

\section{Le point de vue des SU}

De ce fait, bien que les SU mentionnent que les EA apprécient et consultent les travaux des étudiants, il semble que ces derniers indiquent peu (en moyenne un commentaire) ou pas de commentaires écrits. Selon les SU, plusieurs EA qui formulent des remarques le font à l'oral. À cet égard, un SU s'inquiète du fait que des commentaires écrits puissent être lus par une personne autre qu'un des membres de la triade; un autre SU souligne le fait qu'un EA lui a fait part de cette même crainte. Toujours selon les SU, d'autres enseignants font part de leurs observations par écrit en émettant le plus souvent des commentaires courts, de type positif ou d'encouragement.

Le temps requis pour consulter les travaux semble représenter un obstacle pour certains enseignants. Selon deux SU, des EA mentionnent être " en surcharge de travail » alors que d'autres affirment n'avoir tout simplement jamais reçu le lien leur permettant d'accéder aux travaux universitaires de leur stagiaire. En revanche, étant donné qu'un SU accompagne plusieurs stagiaires, il affirme que le portfolio numérique lui amène " moins de travail [et] moins de courriels " puisqu'il n'est pas nécessaire " d'enregistrer des dossiers et de manipuler tous les documents "; tout se retrouve au même endroit.

Mis à part le manque de temps, un SU se questionne sur l'intérêt qu'ont les EA envers les travaux universitaires. D'une part, la plupart des 


\section{REVUE HYBRIDE DE L'ÉDUCATION}

SU s'entendent sur le fait qu'ils sont les « leaders dans les travaux universitaires, donc du portfolio », et qu'ils doivent " prendre l'habitude d'en parler durant les rencontres ». Ils mentionnent également qu'il est nécessaire d'« intégrer graduellement " le portfolio et de " bien communiquer [avec] l'EA pour [son] intégration ». D'autre part, certains SU mentionnent que "la dynamique doit être amenée par l'étudiant ", qu'on doit « responsabiliser les étudiants à montrer [leur] portfolio aux EA » et les inciter à leur communiquer le lien permettant d'accéder au portfolio dès la première prise de contact.

Le point de vue des EA

Les réponses des EA confirment le point de vue des SU quant aux commentaires émis en lien avec les travaux des stagiaires. Six EA disent avoir fait plus de trois commentaires, deux EA en ont fait deux et 11 EA n'en ont rédigé aucun. Quatre EA affirment avoir initié les commentaires avant le SU, alors que la majorité des EA, 15, disent que le SU a rédigé les commentaires en premier. Sept EA confirment que le portfolio améliore la collaboration, six mentionnent qu'il n'entrave ni n'améliore la collaboration et six enseignants n'ont pas répondu à la question. Ils soulignent qu'avec le temps, l'outil sera mieux intégré, ce qui aura comme impact de faciliter encore davantage la collaboration.

Collaborer pour une vision commune afin d'améliorer la qualité de l'accompagnement

Plusieurs travaux, décrits antérieurement, sont déposés sur la plateforme et font l'objet de commentaires oraux ou écrits. Ces travaux visent le développement des compétences du stagiaire.

Le point de vue des SU

Les SU mentionnent que la majorité des étudiants lisent et répondent aux commentaires émis par les formateurs relativement à leurs travaux, et ce, à plusieurs reprises. D'ailleurs, la moitié des SU mentionne que le dispositif permet de faciliter le développement de certaines 


\section{REVUE HYBRIDE DE L'ÉDUCATION}

compétences. Ils affirment, entre autres, que l'analyse réflexive est « un peu plus détaillée " grâce aux commentaires et que les étudiants sont plus rigoureux en ce qui concerne le portrait de classe, lequel semble aussi plus complet : " moins de jugements, moins d'interprétation [...] plus réfléchi ». Le dispositif permet, selon eux, une posture plus professionnelle.

Deux SU affirment que le fait d'avoir accès aux travaux universitaires sur une même plateforme permet d'améliorer le lien avec l'EA et d'avoir une vision commune du stage. De la même façon, un SU soutient que la plateforme Mahara donne l'opportunité aux stagiaires d'être créatifs et qu'il est possible d'observer « [leur] profil et le type d'enseignant qu'ils vont devenir par la couleur, mais aussi par l'organisation » de leur travail. Les SU sont aussi d'accord pour affirmer que le portfolio numérique permet une évaluation plus juste des compétences du stagiaire puisque les commentaires constituent des traces à conserver, par exemple, pour la synthèse finale. Ils considèrent également qu'il serait pertinent de poursuivre l'élaboration du portfolio numérique afin de pouvoir se référer à la collection de travaux du stagiaire en cours de stage, mais aussi pour être en mesure d'aller consulter ce qui a été fait lors des années précédentes.

\section{Le point de vue des EA}

Treize EA ont répondu à la question relative à l'apport du portfolio pour favoriser une vision commune du stage. Huit affirment que cette vision commune est améliorée, trois disent ne pas savoir et deux EA nient une amélioration eu égard à cette vision. Les commentaires des EA mettent en évidence que les rencontres en triade demeurent plus efficaces en ce qui a trait à la collaboration. De façon moins éloquente que les SU, seuls $10 \mathrm{EA}$ s'entendent sur le fait que le dispositif permet de faciliter le développement de certaines compétences; les neuf autres ne le perçoivent pas nécessairement comme un outil aidant à cet égard. Malgré tout, certains commentaires laissés par les EA, dont celui faisant 


\section{REVUE HYBRIDE DE L'ÉDUCATION}

référence à l'accès aux travaux universitaires, lequel permet de questionner plus pertinemment le stagiaire lors de l'évaluation finale, laissent croire que le portfolio permet un accompagnement de la réflexion du futur enseignant.

\section{Discussion}

Les résultats ont permis de dégager deux constats : l'un concerne le portfolio numérique comme outil de médiation et de médiatisation intégrant un dispositif hybride (Charlier et al., 2006) et l'autre est en lien avec les diverses formes de collaboration telles qu'explicitées par Larivée et al. (2006).

Dans un premier temps, cette étude confirme que le portfolio numérique répond au rôle de médiation entre le stagiaire, l'EA et le SU. II permet une meilleure collaboration dans le sens où il est facile pour chacun des acteurs d'accéder aux travaux du stagiaire. Cependant, la médiatisation se fait encore difficilement. En effet, les difficultés en lien avec le partage du lien, le manque d'intérêt et la façon de commenter les travaux des stagiaires mettent en évidence que le processus de scénarisation de contenus sous diverses formes, telles que l'information, l'interaction, la réalisation de tâche, l'autoévaluation, l'accompagnement, etc. (Peraya, 2014), n'est pas encore suffisamment clair pour les différents intervenants. II y a là encore un travail d'approfondissement à faire par rapport à la position de chacun relativement aux travaux universitaires, au leadership de chacun dans la consultation du portfolio, etc.

Dans un deuxième temps, les résultats obtenus permettent de situer le degré de relation, d'engagement et de consensus des deux acteurs clés impliqués dans l'accompagnement du stagiaire. Par le biais du portfolio numérique, l'EA et le SU semblent atteindre le niveau 2 du modèle de Larivée (2003 cité par Larivée et al., 2006) en ce sens que ces deux formateurs se concertent et se coordonnent en vue d'accompagner le stagiaire. Or, dans le Cadre de référence pour la formation des 


\section{REVUE HYBRIDE DE L'ÉDUCATION}

formateurs de stagiaires (Portelance et al., 2008), il est mentionné que le SU doit travailler en concertation avec l'EA en vue d'une formation cohérente du stagiaire, mais qu'il doit aussi développer avec lui un rapport de co-formation nourri à la fois par l'expérience de l'EA et par les fondements théoriques de la formation du futur enseignant que lui-même alimente, d'où sa fonction de "médiateur de savoir " (Gervais, 2008, p. 28). En somme, ce rapport de co-formation exige que le but soit commun et tienne compte des compétences respectives de chaque partenaire (Gervais, 2008), ce qui ne correspond pas exactement aux résultats de la présente étude qui montrent plutôt que les deux acteurs clés se concertent, mais qu'il n'est pas question de partenariat et de coopération. Une recherche menée récemment auprès de SU en enseignement (Cividini, Cody et Truchon Tremblay, soumis) abonde dans le même sens; elle démontre que l'EA et le SU se concertent, mais qu'ils en arrivent rarement, voire même pratiquement jamais, à ce rapport de co-formation qui correspond au niveau 3 du modèle de Larivée (2003 cité par Larivée et al., 2006), lequel se traduit sous la forme d'un partenariat supposant une position d'ouverture aux savoirs et aux expériences des autres (Portelance et al., 2011). Le portfolio numérique pourrait-il contribuer au passage vers cet échelon supérieur dans le modèle de Larivée (2003 cité par Larivée et al., 2006)? Les résultats indiquent que ce dispositif permet d'avoir une vision partagée tout au long du stage. Concrètement, en ayant accès aux travaux universitaires, l'EA peut maintenant donner son opinion sur cette partie de la compétence du stagiaire; quant au SU, il a dorénavant la possibilité de prendre connaissance des commentaires pratiques de l'EA, même s'ils sont peu nombreux, non seulement de façon ponctuelle lors de ses visites à l'école, mais aussi sur une base régulière à distance. L'accompagnement s'organise sous la forme de rencontres espacées dans le temps et «le travail et le cheminement de l'accompagné se font pendant ces temps de rencontre, mais aussi dans l'intervalle » (Prodhomme, 2002), lequel peut être largement exploité par le biais d'un dispositif tel que le portfolio numérique. 


\section{REVUE HYBRIDE DE L'ÉDUCATION}

\section{Conclusion}

Ce texte présente une synthèse des éléments en lien avec la structuration du portfolio numérique dans une visée collaborative de même qu'avec les modalités qui y sont associées. Les résultats montrent certaines difficultés vécues (problèmes techniques, temps requis, définition des rôles quant à l'usage du dispositif, etc.), mais surtout, de nombreux avantages dont la facilité d'appropriation de la structure, la possibilité de laisser des «traces" et l'accessibilité aux travaux universitaires regroupés au même endroit, lesquels permettent aux intervenants d'avoir une vision commune du stagiaire, donc de l'accompagner plus adéquatement, ce qui facilite le développement et l'évaluation de certaines compétences professionnelles.

La recherche tire son originalité du fait qu'à ce jour, peu d'études ont documenté la façon dont l'EA et le SU peuvent exploiter ce dispositif pour mieux accompagner le stagiaire tout en favorisant leur partenariat (Zeichner et Hutchinson, 2004). Elle comporte cependant une limite importante, soit le nombre d'EA qui ont répondu au questionnaire ( $n=$ 19). En fait, ce petit nombre ne permet pas d'obtenir un portrait représentatif des perceptions de cette catégorie d'intervenants, car au total, le projet a mobilisé 58 EA. Est-ce en raison d'un manque d'enthousiasme à l'égard de l'intégration du nouveau dispositif ou encore du choix de la période de l'année (fin du stage), laquelle n'était peut-être pas propice pour remplir le questionnaire? La question se pose... Une seconde limite tient au fait que le portfolio est implanté depuis peu dans le cadre du baccalauréat préscolaire-primaire. Plusieurs difficultés ont donc été soulevées; celles-ci feront l'objet d'amélioration au cours des prochaines années.

Quant aux pistes de recherche et de formation envisagées, nous prévoyons réaliser une analyse des types de commentaires qui favorisent l'accompagnement du stagiaire, plus particulièrement en ce qui concerne

les liens cours-stage. À titre de coresponsables de la formation du 


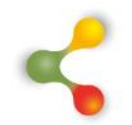

\section{REVUE HYBRIDE DE L'ÉDUCATION}

stagiaire en milieu de pratique, l'EA et le SU doivent le guider dans le développement des 12 compétences (Portelance, 2009) et dans son analyse réflexive (Schön, 1996), particulièrement en l'amenant à établir des liens entre les savoirs formels et expérientiels. Pour ce faire, dans le cadre du processus de supervision, les intervenants doivent réfléchir et dialoguer tout en mettant à profit la complémentarité de leur expertise (Pharand et Boudreault, 2011). Ce segment relatif à la co-formation est en lien avec nos perspectives de formation qui consisteront à faire en sorte d'atteindre le niveau 3 du modèle de Larivée (2003 cité par Larivée et al., 2006). Mais comment atteindre ce troisième niveau, axé sur le partenariat et la coopération, qui permettrait de travailler davantage à une formation intégrative du stagiaire par un meilleur arrimage théoriepratique? Notamment en sensibilisant les EA et les SU à l'importance de reconnaitre le portfolio numérique, non seulement comme un travail universitaire, mais aussi comme l'endroit où s'arriment des savoirs formels et expérientiels. Les EA doivent savoir ce qui est appris à l'université - pour ce faire, les descriptions de cours seront dorénavant intégrées dans le portfolio -, et comprendre qu'en tant que formateurs, il est légitime, voire même nécessaire, qu'ils accèdent aux données du portfolio, sans avoir une «impression de voyeurisme »! Tout cela, une fois que les deux catégories de formateurs se seront réellement approprié la structure du dispositif, car actuellement, tous estiment qu'il est trop tôt pour qu'ils puissent se centrer pleinement sur sa visée collaborative. II faudra du temps, mais les résultats laissent croire qu'il y a là un réel potentiel en termes de collaboration entre les deux intervenants; une collaboration qui sera renforcée au cours de la prochaine année par la participation des membres du corps professoral, lesquels seront invités à intégrer le portfolio numérique dans le cadre de leurs cours, permettant ainsi de tendre encore davantage vers une approche-programme... 


\section{REVUE HYBRIDE DE L'ÉDUCATION}

\section{Références}

Anadon, M. (dir.) (2007). La recherche participative. Multiples regards. Québec, QC : Presses de l'Université du Québec.

Bardin, L. (1989). L'analyse de contenu (5e éd.). Paris, France : Presses universitaires de France.

Bibeau, R. (2007). À chacun son portfolio numérique. Clic, Bulletin collégial des technologies de l'information et des communications, $65,1-9$.

Charlier, B., Deschryver, N. et Peraya, D. (2006). Apprendre en présence et à distance. Une définition des dispositifs hybrides. Distances et savoirs, 4, 469-496.

Cividini, M., Cody, N. et Truchon Tremblay, C. (soumis). Entre compétences prescrites et réelles du superviseur: quelles stratégies pour accompagner les stagiaires?

Cody, N. (2013). La formation initiale d'un enseignant au préscolaireprimaire. Une interaction constante entre les activités théoriques, pratiques et réflexives. Thèse de doctorat. Allemagne: Presses académiques francophones.

Dionne, L. et Savoie-Zajc, L. (2011). Sens, caractéristiques et retombées de la collaboration entre enseignants et contribution au développement professionnel. Dans L. Portelance, C. Borges et J. Pharand (dir.), La collaboration dans le milieu de l'éducation. Dimensions pratiques et perspectives théoriques (p. 45-60). SainteFoy, QC : Presses de I'Université du Québec.

Gagnon, C., Mazalon, É., et Rousseau, A. (2010). Fondements et pratique de l'alternance en formation à l'enseignement professionnel : quelques données de recherche autour de l'élaboration et de la 


\section{8}

\section{REVUE HYBRIDE DE L'ÉDUCATION}

mise en œuvre à l'Université de Sherbrooke. Nouveaux cahiers de la recherche en éducation, 13(1), 21-41.

Gervais, C. (2008). Pour une formation des stagiaires en concertation. Dans M. Boutet et J. Pharand (dir.). L'accompagnement concerté des stagiaires en enseignement (p. 91-108). Québec, QC : Presses de l'Université du Québec.

Goupil, (1998). Portfolios et dossiers d'apprentissage. Montréal, QC : Chenelière/McGraw-Hill.

Labelle, K. (2015). Étude de cas d'entretiens sur le portfolio favorisant la pratique réflexive chez des étudiants en éducation préscolaire et en enseignement primaire. Mémoire de maitrise. Montréal, QC : Université du Québec à Montréal.

Larivée, S.J., Kalubi, J.-C. et Terrisse, B. (2006). La collaboration écolefamille en contexte d'inclusion: entre obstacles, risques et facteurs de réussite. Revue des sciences de l'éducation, 32(3), 525-543.

Martin, D. (2002). Terrain et théories dans les réformes de la formation des maitres. Dans M. Carbonneau et M. Tardif (dir.), Les réformes en éducation, leurs impacts sur l'école (p. 95-109). Sherbrooke, QC : Éditions du CRP.

Naccache, N., Samson, L. et Jouquan, J. (2006). Le portfolio en éducation des sciences de la santé : un outil d'apprentissage, de développement professionnel et d'évaluation. Pédagogie médicale, 7(2), 110-127.

Peraya, D. (2014). Regards sur les formes de médiatisation de la formation et de l'apprentissage. Synergies Pays Germanophones, (7), 19-32. Repéré à http://archive-ouverte.unige.ch/unige:45054

Peraya, D. (2010). Médiatisation et médiation. Des médias éducatifs aux ENT. Dans V. Liquète (dir.), Médiations (p. 33-48). Paris, France : CNRS. Repéré à http://archive-ouverte.unige.ch/unige:12312 


\section{$\&$}

\section{REVUE HYBRIDE DE L'ÉDUCATION}

Perrenoud, P. (2001). Développer la pratique réflexive dans le métier d'enseignant. Paris, France : Édition sociale française.

Petit, M. (2016). Caractéristiques d'une supervision à distance de stagiaires en enseignement en ce qui concerne la création d'un sentiment de présence. Revue internationale de pédagogie de l'enseignement supérieur, 32(1). Repéré à http://ripes.revues.org/1041

Pharand, J. et Boudreault, P. (2011). Enseignants associés et superviseurs. Perceptions des uns à l'égard des autres et collaboration réciproque. Dans F. Guillemette et M. L'Hostie (dir.), Favoriser la progression des stagiaires en enseignement (p. 121141). Québec, QC : Presses de l'Université du Québec.

Portelance, L., Pharand, J., et Borges, C. (2011). Mieux comprendre la collaboration pour mieux collaborer. Dans L. Portelance, C. Borges et J, Pharand (dir.), La collaboration dans le milieu de l'éducation (p. 215-224). Québec, QC : Presses de l'Université du Québec.

Portelance, L. (2009). Élaboration d'un cadre de référence pour la formation des enseignants associés québécois. Éducation et francophonie, 37(1), 26-49.

Portelance, L., Gervais, C., Lessard, M. et Beaulieu, P. (2008). La formation des enseignants associés et des superviseurs universitaires. Cadre de référence. Québec, QC : MELS.

Prodhomme, M. (2002). Accompagnement de projet professionnel et bilan de compétences. Être là et s'abstraire pour permettre au sujet d'advenir. Éducation Permanente, 153(4), 79-89.

Schön, D. A. (1996). À la recherche d'une nouvelle épistémologie de la pratique et de ce qu'elle implique pour l'éducation des adultes. Dans J.-M. Barbier (dir.), Savoirs théoriques et savoirs d'action ( $p$. 201-222). Paris, France : Presses Universitaires de France. 


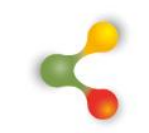

\section{REVUE HYBRIDE DE L’ÉDUCATION}

Zeichner, K. et Hutchinson, E. (2004). Le rôle du portfolio de l'enseignant comme outil pour identifier et développer les compétences des enseignants. Recherche et formation, 47, 69-78.

Wolf, K. (1996). Developing an Effective Teaching Portfolio. Improving Professional Practice, 53(6), 34-37. 